\title{
The bi- (or multi-) phasic response of cardiac remodelling to endurance exercise related to the article: 'From talented child to elite athlete: The development of cardiac morphology and function in a cohort of endurance athletes from age I 2 to 18' by Bjerring and colleagues
}

European Journal of Preventive Cardiology $0(0)$ I-3 (C) The European Society of Cardiology 2020 (c) (1) ()

Article reuse guidelines: sagepub.com/journals-permissions DOI: 10.1 I77/2047487320929245 journals.sagepub.com/home/cpr (S)AGE

\author{
Martin Burtscher
}

In a recent issue of this journal, Bjerring and colleagues present unique cardiac adaptations derived from longitudinal observations in young talented cross-country skiers. ${ }^{1}$ They report eccentric remodelling from 12-15 years followed by concentric remodelling from 15-18 years. These findings contribute to the contemporary understanding of cardiac development during growth of talented endurance athletes. Although myriads of studies have dealt with cardiac adaptive responses to various types of sports at different ages, only limited data are available from longitudinal investigations, in particular during growth and maturation of children and adolescents.

\section{From past to present}

When talking about cardiac morphology in crosscountry skiers, reference must be made to the early work of Salomon Henschen (1899) who may be considered as the 'father of the athlete's heart', defining respective characteristics based on systematic physical examination (percussion) of cross-country skiers. ${ }^{2} \mathrm{He}$ described symmetric cardiac enlargement (dilatation and hypertrophy - eccentric remodelling) in those athletes and suggested that dilatation would occur first followed by increasing wall thickness. Particularly noteworthy, Henschen already proposed those changes to be physiological (and beneficial) adaptive responses to training associated with improved exercise performance.

More than four decades ago, Morganroth and colleagues suggested different types of cardiac remodelling, the eccentric type in endurance athletes and the concentric type in strength athletes. ${ }^{3}$ More recently, others reported different cardiac adaptations in various endurance sports, i.e. cycling, marathon running and triathlon, triggered by distinct exercise-related pressure and volume loads. Beside the most prevalent eccentric left ventricular (LV) remodelling (particularly in cyclists) intermediate forms and even concentric remodelling in a minority of highly-trained endurance athletes were shown. ${ }^{4}$ It was pointed out that both the process of dilatation and increasing ventricular mass start at an early age and continue throughout the athlete's career. Bjerring et al. have complemented the understanding of the dynamic cardiac development during maturation of talented athletes by demonstrating a specific shift between different forms of remodelling; also providing some insight in potential interaction between growth and training. ${ }^{1,5,6}$

\section{Effects of growth on cardiac development}

Obviously, growth has a major impact on cardiac morphology, simply due to the fact that blood supply to working muscles is closely related to body mass. This is also reflected by the reported increases of both body mass and LV mass by about $50 \%$ from $12-15$ years in athletes followed by Bjerring et al. ${ }^{1,6}$ Findings from a longitudinal investigation including 125 children and adolescents of both sexes suggest that changes in LV mass during puberty are best predicted by normal

\footnotetext{
University of Innsbruck, Austria
}

\section{Corresponding author:}

Martin Burtscher, University of Innsbruck, Department of Sport Sciences, Fürstenweg 185, A-6020 Innsbruck, Austria.

Email: martin.burtscher@uibk.ac.at 
growth and the associated lean body mass (LBM). ${ }^{7}$ Striking changes during pubertal growth are not only typical for height and body mass but also for blood volume (BV) and systemic blood pressure, which may influence cardiac morphology and seems to be more pronounced in males than females. ${ }^{8} \mathrm{BV}$ increases linearly with LBM similarly in children, adolescents and adults of both sexes, however, the rise in BV proceeds slightly faster than the one of LBM during puberty. The question arises (and has been addressed by Bjerring and colleagues) how effects of normal growth and maturation on cardiac development in talented athletes are modulated by continuing regular intense endurance training from child- to adulthood.

\section{Effects of talent (genetics) and training on cardiac remodelling}

Whether cardiac adaptation to endurance training, as demonstrated in athletes, is primarily modulated by genetic predisposition or by training stimuli has been investigated in a one-year intense endurance training study including young (adult) sedentary males and females. ${ }^{9}$ Cardiac morphology and function were assessed every 3 months by means of cardiac magnetic resonance imaging and right heart catheterization. Unexpectedly, but similar to the findings of Bjerring et al., the authors observed a biphasic response to training. An initial increase in the LV mass without change in volume (concentric hypertrophy) was followed after 6-9 months of training by elevation of the left ventricular end-diastolic volume (LVEDV), re-establishing the baseline mass-to-volume ratio (eccentric hypertrophy). The initial concentric hypertrophy was considered as an appropriate response to the lower intensity during the first training period and that of eccentric hypertrophy to the subsequent higher training intensity. ${ }^{9}$ A two-fold conclusion can be derived from that study. First, a prolonged and appropriate training stimulus is the primary determinant for cardiac adaptation and, second, both concentric and eccentric adaptive responses may occur in a biphasic manner. Although these findings indicate similar adaptations in cardiac morphology and function as known from elite athletes, the aerobic capacity was far from those in elite athletes, which can be attributed to the duration of training and certainly also to genetic components of the individual trainability. This is underpinned by the fact that maximal oxygen uptake values as high as $90 \mathrm{ml} / \mathrm{min} / \mathrm{kg}$ achieved by the world's top cross-country skiers can never be reached by individuals who are not optimally gifted. ${ }^{10}$ Moreover, cardiac remodelling also depends on the modality (and intensity) of exercise, i.e. higher pressure-load (static component) favouring concentric and higher volume-load (dynamic component) favouring eccentric adaptation. Accordingly, athletes are mostly exposed to a 'mixed' cardiovascular load; for example, cross-country skiing (classic technique) can be characterised by low static and high dynamic components and skating technique by moderate static and high dynamic components, ${ }^{11}$ potentially influencing cardiac development demonstrated by Bjerring and colleagues. ${ }^{1,6}$

\section{Synergistic effects of growth and systematic endurance training on cardiac remodelling}

How the increasing cardiac enlargement with chronological age (6-18 years) is modulated by exercise training was recently evaluated in a large meta-analysis. ${ }^{12}$ As a consequence of regular endurance exercise, young athletes clearly had more pronounced LV enlargement than non-athletes, and these changes were larger during pubertal growth. Males showed a greater LV hypertrophic response than females, likely due to hormonal differences. This meta-analysis also revealed a greater prevalence of training-related and training-unrelated electrocardiogram changes (e.g. inverted $\mathrm{T}$ waves) in athletes compared to non-athletes, an effect similar to adult athletes. From those analyses, the complex interaction between growth, maturation and exercise training becomes apparent and has been invaluably complemented by the follow-up study by Bjerring et al. ${ }^{1,6}$ Whereas parameters of growth (anthropometric measures) did not considerably differ between continuously active athletes (primarily cross-country skiers) and those who terminated their active career between 12-18 years, cardiac morphology and associated aerobic capacity did. After initial concentric remodelling at the age of 12 years, active athletes showed (in contrast to the less active) dilatation of cardiac chambers and reduction of the relative wall thickness to baseline (eccentric remodelling) followed by concentric remodelling between the age of 15-18 years. The two groups primarily differed with regard to training volume (and intensity). Combined data analyses of both groups revealed that LV mass increased by $3.6 \mathrm{~g}$ per weekly hour of endurance exercise between $12-15$ years and by $6.4 \mathrm{~g}$ between $15-18$ years, whereas the LVEDV increased by $2.3 \mathrm{ml}$ per weekly hour of endurance training only from $12-15$ years. Presumably, the shifts between concentric, eccentric and again concentric remodelling result from interactions between growth, maturation and the training-related stimuli of pressure and volume (over)load. Certainly, the final stage of athletic 
development is not yet reached, thus further follow-up of this cohort to the age of peak performance will be of particular interest.

\section{What do we know, where should we go?}

The athlete's heart exhibits specific morphological and functional characteristics as a consequence of regular exercise training, primarily depending on the type, volume and intensity of the exercise performed. Systematic engagement in intense endurance exercise provokes dilatation of all chambers and, in contrast to pathological conditions, a symmetric increase in wall thickness. Evidence for distinct phases of cardiac remodelling during growth and maturation has been substantiated by the findings of Bjerring and colleagues. However, the precise number, extent and duration of such phases from child- to adulthood remain to be elucidated, but are very likely modulated by individual responses to year-long training schedules. Future longitudinal studies performed in interdisciplinary teamwork are needed to fully understand age-, sexand training-dependent conditions and mechanisms of physiological (but also pathological) cardiac remodelling in talented athletes.

\section{Declaration of conflicting interests}

The author(s) declared no potential conflicts of interest with respect to the research, authorship and/or publication of this article.

\section{Funding}

The author(s) received no financial support for the research, authorship and/or publication of this article.

\section{References}

1. Bjerring AW, Landgraff HE, Leirstein S, et al. From talented child to elite athlete: The development of cardiac morphology and function in a cohort of endurance athletes from age 12 to 18. Eur J Prev Cardiol. Epub ahead of print 28 April 2020; DOI: 10.1177/2047487320921317.

2. Henschen S. Skilanglauf und Skiwettlauf: Eine medizinische Sportstudie. Mitt Med Klin Upsala (Jena) 1899; 2: $15-18$.

3. Morganroth J, Maron BJ, Henry WL, et al. Comparative left ventricular dimensions in trained athletes. Ann Intern Med 1975; 82: 521-524.

4. Hoogsteen J, Hoogeveen A, Schaffers H, et al. Myocardial adaptation in different endurance sports: An echocardiographic study. Int $J$ Cardiovasc Imaging 2004; 20: 19-26.

5. Bjerring AW, Landgraff HE, Leirstein $\mathrm{S}$, et al. Morphological changes and myocardial function assessed by traditional and novel echocardiographic methods in preadolescent athlete's heart. Eur J Prev Cardiol 2018; 25: 1000-1007.

6. Bjerring AW, Landgraff HE, Stokke TM, et al. The developing athlete's heart: A cohort study in young athletes transitioning through adolescence. Eur J Prev Cardiol. 2019; 26: 2001-2008.

7. Janz KF, Dawson JD and Mahoney LT. Predicting heart growth during puberty: The Muscatine Study. Pediatrics 2000; 105: E63.

8. Shankar RR, Eckert GJ, Saha C, et al. The change in blood pressure during pubertal growth. $J$ Clin Endocrinol Metab 2005; 90: 163-167.

9. Arbab-Zadeh A, Perhonen M, Howden E, et al. Cardiac remodeling in response to 1 year of intensive endurance training. Circulation 2014; 130: 2152-2161.

10. Burtscher M, Nachbauer $\mathrm{W}$ and Wilber R. The upper limit of aerobic power in humans. Eur J Appl Physiol 2011; 111: 2625-2628.

11. Mitchell JH, Haskell W, Snell P, et al. Task Force 8: Classification of sports. J Am Coll Cardiol 2005; 45: 1364-1367.

12. McClean G, Riding NR, Ardern CL, et al. Electrical and structural adaptations of the paediatric athlete's heart: A systematic review with meta-analysis. Br J Sports Med 2018; 52: 230. 